\title{
Risiko Thrombose: 60 Jahre Pille - was sind die Fakten?
}

Anlässlich des 7. Weltthrombosetages am 13.10.2020 veranstaltete das Aktionsbündnis Thrombose eine virtuelle Podiumsdiskussion, um die Entwicklung der medikamentösen Kontrazeption in den letzten 60 Jahren und das Thromboserisiko unter hormoneller Kontrazeption zu diskutieren. Unter professioneller Moderation des Journalisten Henning Quanz wurden erstmalig der WTT und die bewährte Podiumsdiskussion als Streaming-Format ins Netz gestellt. Eingeleitet mit einer Befragung von Passanten auf der Straße zu ihrer Einstellung zur und Erfahrung mit der Pille diskutierten die Podiumsteilnehmer Prof. Dr. Rupert Bauersachs, Darmstadt, und Prof. Petra Stute, Bern, im Studio mit den zugeschalteten Teilnehmern Prof. Ludwig Kiesel, Münster, Dr. Susanne Hampel, Berlin, Dr. Hannelore Rott, Duisburg, und PD Dr. Mareike Lankeit, Berlin. Seit 1963 sei bekannt, dass Zusammenhänge zwischen Hormonen und Thromboembolien beständen. In den aktuellen Leitlinien zur hormonellen Empfängnisverhütung werde erneut darauf hingewiesen, dass GestagenMonopräparate in der Verordnung bevorzugt werden sollen und dass vor der Verordnung die Frauen ausführlich über die Risiken des „Medikaments Pille“ aufzuklären seien. Ferner wurde darauf hingewiesen, dass die Symptome einer Thrombose oder Lungenembolie von den Pillenanwenderinnen, aber auch von den involvierten Ärzten/Ärztinnen häufig ignoriert und zu spät erkannt würden mit fatalen Folgen für die Betroffenen. Auch hier wurde Aufklärungsbedarf gesehen. Einigkeit bestand beim Podium wie auch bei den befragten Passanten dahingehend, dass die Zulassung der „Pille“ vor 60 Jahren, trotz aller Kritik, für die Frauen und ihre Selbstbestimmung ein Segen war.

Als Abschluss der Veranstaltung ließ es sich der virtuelle Rudolf Virchow nicht nehmen, dem diesjährigen Virchow-Preisträger Prof. Frederikus Klok aus den Niederlanden zu dem mit $5000 €$ dotierten Preis zu gratulieren. Prof. Klok wurde ausgezeichnet für seine frühzeitigen Forschungsarbeiten zur erhöhten Gerinnungsaktivierung von COVID-19, den dadurch vermehrt auftretenden thromboembolischen Ereignissen bei COVID-19-Patienten und einer damit erforderlichen Anpassung der Thromboseprophylaxe bei diesen Patienten.

Das Streaming-Format hat sich als so erfolgreich erwiesen, dass das Aktionsbündnis durchaus in Erwägung ziehen wird, in der Zukunft dabei zu bleiben.

Dr. Jutta Schimmelpfennig AG Thrombose DGP

Aktionsbündnis Thrombose

\section{IMPRESSUM}

Verantwortlich für Mitteilungen der DGP:

Dr. med. Erika Mendoza 